\title{
Dark Matter-Like Effects in Spiral Galaxies Caused by Intense Isotropic Background Radiation of Ultralong Wavelength Photons
}

\author{
Christoph Schultheiss ${ }^{1}$ \\ ${ }^{1}$ Karlsruhe Institute of Technology (KIT), Institute for Pulsed Power and Microwave Technology (IHM), P.O. \\ Box 3640, 76021 Karlsruhe, Germany \\ Correspondence: Christoph Schultheiss, Karlsruhe Institute of Technology (KIT), Institute for Pulsed Power and \\ Microwave Technology (IHM), P.O. Box 3640, 76021 Karlsruhe, Germany. E-mail: fc0695@partner.kit.edu
}

Received: May 14, 2015 Accepted: June 11, 2015 Online Published: December 3, 2015

doi:10.5539/apr.v8n1p7 URL: http://dx.doi.org/10.5539/apr.v8n1p7

\begin{abstract}
The rotation anomaly of outer branches of spiral galaxies can be explained on the basis by a gravitational theory published in 2008. It is based on the interaction of charges with electromagnetic radiation with ultralong wavelengths which were generated by the Big Bang and which are densely distributed in the expanding space. The cross section of the interaction of charges with these waves depends on the Rayleigh scattering governed by the well known $\omega / \omega_{p}$ - factor to the fourth, where $\omega$ is the frequency of the wave and $\omega_{\mathrm{p}}$ the "natural frequency" of the particle. Natural frequency means that charges respectively matter oscillate with higher frequency. The $\omega / \omega_{p}$-factor implies an increase of the gravitation constant with increasing length of path in space and gives a possible explanation of why stars in outer spirals move with higher orbital velocity.
\end{abstract}

Keyword: dark matter, electromagnetic theory of gravitation, Rayleigh scattering, rotation anomaly, spiral galaxies

\section{Introduction}

It is an observational fact that in most of the spiral galaxies the outer spiral branches move with higher velocity than the inner branches (Sofue \& Rubin, 2001; Sofue, 2013). The gravitational law of visible masses predicts an exactly opposite velocity profile. For instance, the galactic orbital velocity of the sun is not $160 \mathrm{~km} / \mathrm{s}$ (as determined by the masses of visible stars inside the sun's orbit) but is more than $200 \mathrm{~km} / \mathrm{s}$ (Reid, Readhead, Vermeulen, \& Treuhaft, 1999).

This observation necessitated the postulation of dark matter, which is assumed to be densely distributed near the outer branches of the spiral galaxies and responsible for the increased velocity (Freeman, 1970; Persic \& Salucci, 1970). Up to now, there has been no direct experimental verification for the existence of dark matter. In principle this is a complicated task, because the lack of electromagnetic interaction restricts the possible detection methods to weak and indirect ones (Bergstrom, 2000).

But there is also a classical way to understand this phenomenon. The main content of space is electromagnetic zero-point energy, as described by Casimir in 1948. In addition, a small fraction of thermal photons, called ultralong-wavelength photons (ULW), are proposed to be responsible for the gravitational attraction of masses by means of mutual screening as described by Schultheiss (2008). The advantage of this proposal is that it does not require new physical hypotheses such as the assumed interaction with hitherto undetected dark particles. The same is true for dark energy (Kolb \& Turner, 1990), which can be interpreted to result from the pressure of the ULW-photons with maximum strength at the edge (rim) of space. Here the counter pressure of ULW-photons coming from outside vanishes.

But what are the main tenets of this gravitational theory? The original idea goes back to a historic theory of La Sage and others (Aronson, 1964). They understand gravitation as an inward-directed pressure on masses generated by the friction of an isotropic flux of fast hypothetic particles. But this approach also implied a deceleration of these particles and heat development caused by friction in the masses, two phenomena that have not been observed in studies of gravitation. In the present paper, I propose a way around these problems and suggest that ULW photons are the "hypothetic particles" described by La Sage and others. 


\section{Main Features of the Attraction of Charges Caused by Mutual Screening of ULW-Photons}

The basic idea presented in Schultheiss (2008) is to assume a very dense isotropic flux $\mathrm{j}$ of a spectrum of thermal ULW photons in the space with wavelengths $\lambda$ up to $10^{26} \mathrm{~m}$, comparable to the diameter of space (Gott III, 2005). The dimension of $\mathrm{j}$ is the number of ULW-photons per unit time. These photons penetrate an interaction sphere $\mathrm{F}_{\mathrm{p}}$ of a charge, and reflect or scatter from it (see Figure 1). The interaction takes place with a probability corresponding to the Rayleigh cross section (Feynman, 1965) (Note that the derivation of the formula is well known in literature):

$$
\sigma_{p}=\pi r_{p}^{2}=\frac{8}{3} \pi r_{0}^{2} \cdot \frac{\omega^{4}}{\left(\omega^{2}-\omega_{p}^{2}\right)^{2}}
$$

The radius $r_{0}=2.8 \cdot 10^{-15} \mathrm{~m}$ is the classical electron radius. $\omega_{\mathrm{p}}$ is the so called "natural frequency" for the case that the frequencies of the charged particle oscillations are higher than the frequency $\omega$ of the ULW-waves (the $\omega$-factor in Equation 1 is also responsible for scattering of visible light by air molecules which leads to the blueness of the sky). For ULW photons, one can assume that $\omega_{p}>\omega$. Therefore, the $\omega$-term in the cross section in Equation 1 can be very small in value.

The radius of the charged particle is in the order of $r_{0}$, the particle interaction radius $r_{p}$ derived from the cross section is much smaller because of the quadratic $\omega$-term:

$$
r_{p}=2 \sqrt{\frac{2}{3}} r_{0} \frac{\omega^{2}}{\omega_{p}^{2}}
$$

The resulting interaction sphere area of the particle is:

$$
F_{p}=4 \pi r_{p}^{2}=\frac{32}{3} \pi r_{0}^{2} \frac{\omega^{4}}{\omega_{p}^{4}}
$$

As can be seen in Figure 1, before the isotropic photon flux $\mathrm{j}$ enters the particle interaction sphere $\mathrm{F}_{\mathrm{p}}$, it passes through the outer shell area $F_{s}$ in a nearly normal direction because the quotient $r_{p} / R$ is generally small. The particle at the outer sphere scatters with the Rayleigh cross section $\sigma_{p}$ mentioned above. Hence, the flux $j$ via the center particle is reduced by the quantity of the scattered flux $\Delta \mathrm{j}$, which is governed by the area factor $\sigma_{\mathrm{p}} / \mathrm{F}_{\mathrm{s}}$ :

$$
\Delta j=j \frac{\sigma_{p}}{F_{S}}=\frac{2}{3} j \frac{r_{0}^{2}}{R^{2}} \frac{\omega^{4}}{\omega_{p}^{4}}
$$

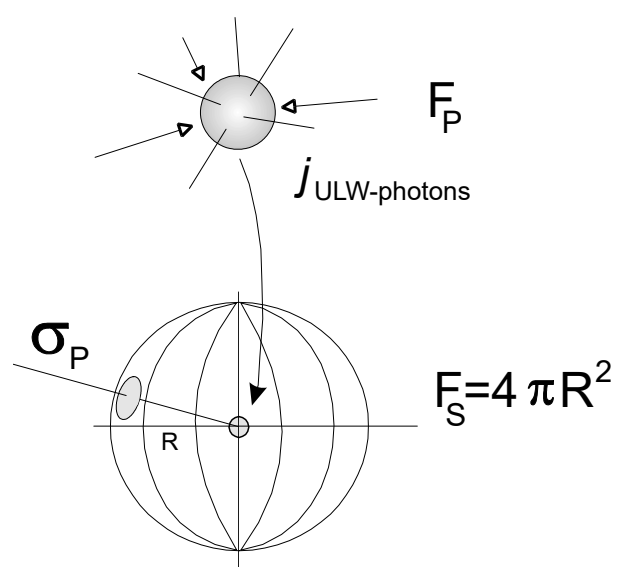

Figure 1. An isotropic flux $\mathrm{j}$ of penetrating ULW photons enters the interaction sphere $F_{p}$ of a charged particle located at the center of a larger sphere with radius $\mathrm{R}$ and interacts with it. A second charged particle located at the larger sphere with cross section $\sigma_{\mathrm{p}}$ screens photons from the center particle and a virtual attraction force will appear (Figure 1 and legend taken from Schultheiss (2008)) 
The reduced flux $j-\Delta j$ appears at the center of the interaction sphere, where the screening particle is located. In the opposite direction the flux $\mathrm{j}$ is unchanged. Thus, the center particle experiences an overshooting force caused by $\Delta \mathrm{j}$ via the screening particle. Since the center particle itself screens the flux to the outer sphere particle with the same probability, a virtual mutual central force between both particles appears.

Note that the cross section in Equation 4 is generally tiny, so that accumulations of matter such as stars are permeated by ULW-photons as easily as dust particles by long-wavelength radio waves.

The thesis of Schultheiss (2008) is that the virtual mutual force $f_{\text {virtual }}$ (Equation 4) with its $\left(\omega / \omega_{p}\right)^{4}$ dependence is, for example, equal to the gravitational force between two electrically neutral hydrogen atoms $\mathrm{m}_{\mathrm{H}}$ separated by the distance $\mathrm{R}$ :

$$
\mathrm{f}_{\text {virtual }}=2 \cdot \tau \cdot \Delta \mathrm{j} \frac{2 \cdot \mathrm{h}}{\lambda}=\frac{8}{3} \tau \cdot \mathrm{j} \cdot \frac{\mathrm{r}_{0}^{2}}{\mathrm{R}^{2}} \frac{\omega^{4}}{\omega_{\mathrm{p}}^{4}} \frac{\mathrm{h}}{\lambda}=\mathrm{f}_{\text {grav }}=\mathrm{g} \cdot \frac{\mathrm{m}_{\mathrm{H}}^{2}}{\mathrm{R}^{2}}
$$

The virtual force $f_{\text {virtual }}$ in Equation 5 (left) generated by ULW photons contains the factor 2 because matter always appears to be electrically neutral and electrons have the same magnitude of charge as protons. The sign of the charges is opposite but this does not matter. That the mass of electrons is a factor of 1836 smaller than the proton mass plays has little significance in this context, because in a strong gravitational field a weak electrical polarisation between protons and electrons should appear. In Equation 5, a second factor 2 appears because the optimum momentum transfer at a reflection of a ULW-photon is $2 \cdot \mathrm{h} / \lambda$. To take all other scattering angles (with lower momentum transfer) into account the factor $\tau<1$ in Equation 5 corresponds to the total scattering cross section.

Note that even photons with cosmic wavelengths can be scattered by a single electron or proton as well as by a single neutron which in this context can be considered as a composite of proton and electron. Following the rules of Quantum Electrodynamics (QED) even photons can be scattered by other photons (Kanda, 2011) (this applies to all wavelengths). The following consideration may allow an easy access to this kind of physics: As a result of the quantum-mechanical uncertainty principle, photons can transmute to a virtual fermion/anti-fermion pair. Then the ULW-photon flux couple to both of these virtual charged particles (masses). The decisive issue is that this process takes place with the duty cycle of $\Delta \mathrm{t} \cdot v \geq \mathrm{hv} / \Delta \mathrm{E}$, where $\Delta E \cong m_{e} c^{2}$. For visible light the value is approximately $\Delta t \cdot v \geq 10^{-6}$ - that is, most of the time the photon path is straight (mean free path $\approx \lambda=10^{-6} \mathrm{~m}$ ), and briefly interrupted by photon-ULW-photon scattering which leads to a polygonal path. The deflection develops like $a t^{2}$ while for example a continuous Newtonian increase of deflection follows $\frac{1}{2} a t^{2}$. It is interesting at this point to note that Einstein, in his Theory of General Relativity (Einstein, 1916), at first assumed a Newtonian deflection of light. But measurements hint to a higher value of about a factor of two. A correction of his theory is described in (Klein, 1996).

Schultheiss (2008) could demonstrate how ULW photons could be generated in the context of the Big Bang (Lemaitre, 1931). In this Compton-collision framework a photon reflects back and forth between a fixed and movable mirror (i.e., charges). It accelerates the movable mirror in such a manner that the increase of path and wavelength develops congruently - an effect which is called "congruence between path and wavelength". This process was exactly derived based on the rules of the Theory of Special Relativity, as will be sketched out in the following:

\section{Congruence between Path and Wave}

The functional relationship between mirror distance and wave train length is shown in the Minkowski space-time presentation of Figure 2. The left graph shows the wave train path (shown as an arrow) in the fixed mirror system $\mathrm{S}$ and the right graph shows the wave train path in the movable mirror system $\mathrm{S}^{\prime}$. If the wave train arrow in one system is horizontally aligned (wave train head and tail arrive simultaneously), then in the other system the wave train arrow is slanted. The coordinates of the laboratory system S in Figure 2 are transformed into the system $\mathrm{S}^{\prime}$ of the movable mass $\mathrm{m}$ by means of the Lorentz transformation $c t^{\prime}=\gamma \cdot(c t-\beta x)$ and $x^{\prime}=\gamma \cdot(x-\beta c t)$. The transformation relates to the common origin, where the $c t$-axis meets the $c t^{\prime}$-axis. This point is in the past at the virtual time $c t_{0}=-L / \beta$. Therefore, before the transformation all coordinates in Figure 1 should be rewritten (see Equation 6). Figure 2 and legend is taken from Schultheiss (2008). 

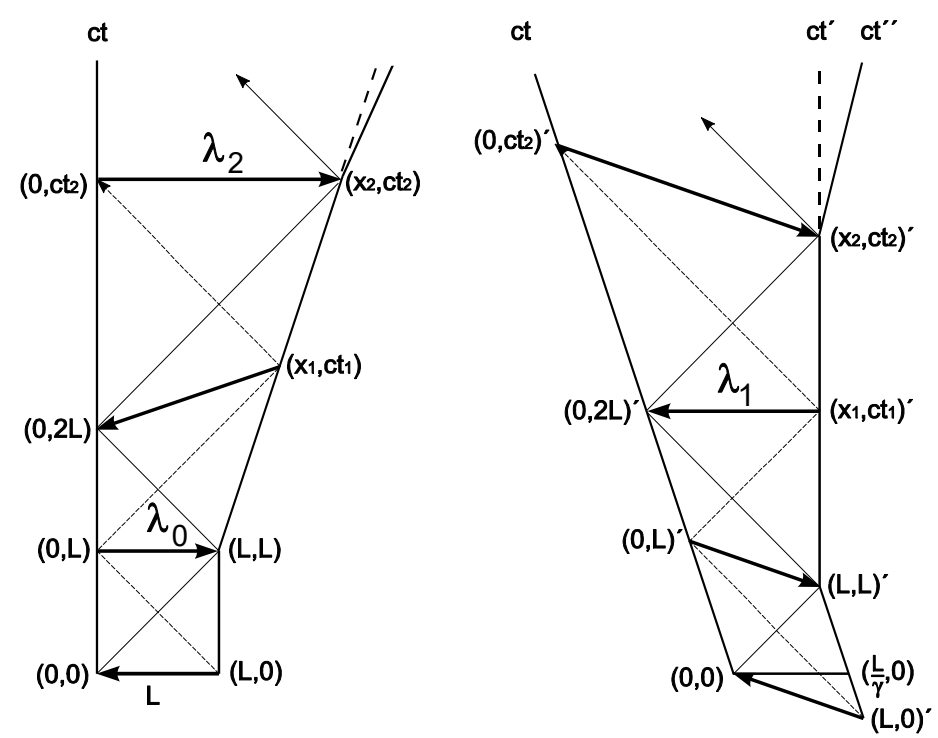

Figure 2. Space-time presentation of $180^{\circ}$-Compton collisions of a photon between a fixed- (ct-axis) and a movable mirror (ct'-axis). The wave train arrow is alternately horizontal- $\left(\lambda_{0}, \lambda_{1}\right.$ and $\left.\lambda_{2}\right)$ or slanted

Let $\beta \cdot c$ be the velocity of a $180^{\circ}$ Compton collision then the transformed coordinates are:

$$
\begin{gathered}
(0, L)^{\prime} \rightarrow\left(0, \frac{L}{\beta}\right)^{\prime}=\left[-\gamma L, \gamma \frac{L}{\beta}\right] \\
(L, L)^{\prime} \rightarrow\left(L, \frac{L}{\beta}\right)^{\prime}=\left[0, \gamma \frac{L}{\beta}\left(1-\beta^{2}\right)\right] \\
(0,2 L)^{\prime} \rightarrow\left(0, \frac{L}{\beta}(1+\beta)\right)^{\prime}=\left[-\gamma L(1+\beta), \gamma \frac{L}{\beta}(1+\beta)\right] \\
\left(x_{1}, c t_{1}\right)^{\prime}=\left(L \frac{1}{1-\beta}, \frac{L}{\beta} \frac{1}{1-\beta}\right)^{\prime}=\left[0, \gamma \frac{L}{\beta}(1+\beta)\right] \\
\left(x_{2}, c t_{2}\right)^{\prime}=\left(L \gamma^{2}(1+\beta)^{2}, \frac{L}{\beta} \gamma^{2}(1+\beta)^{2}\right)^{\prime}=\left[0, \gamma \frac{L}{\beta}(1+\beta)^{2}\right] \\
\left(0, c t_{2}\right)^{\prime}=\left(0, \frac{L}{\beta} \gamma^{2}(1+\beta)^{2}\right)^{\prime}=\left[-L \gamma^{3}(1+\beta)^{2}, \frac{L}{\beta} \gamma^{3}(1+\beta)^{2}\right]
\end{gathered}
$$

The following expressions for the wave-train lengths and path lengths are identical to the length of Doppler shifted waves:

$$
\begin{aligned}
& \lambda_{0}=(L, L)-(0, L),=L \\
& \lambda_{1}=-(0,2 L)^{\prime}+\left(x_{1}, c t_{1}\right)^{\prime}=L \gamma(1+\beta) \\
& \lambda_{2}=\left(x_{2}, c t_{2}\right)-\left(0, c t_{2}\right)=L \gamma^{2}(1+\beta)^{2} \quad \text { etc. }
\end{aligned}
$$


The scheme in Equation 7 demonstrates that just when the wave head touches the opposite mirror, the mirror separation and the horizontally aligned wave train path are exactly equal in length. In the subsequent collisions

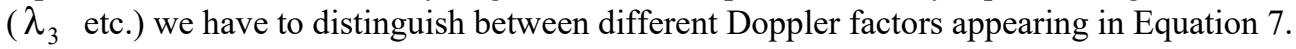

Equation set 7 can be called "congruence equations". With increasing mirror distance, the photon momentum decreases but will not vanish in this framework, even for cosmic separations. In this sense, the number of photons is conserved. A further transfer of energy from ULW photons onto particles is only possible if the mirrors and waves continue expansion. Note that a thermalization of ULW photon energy in this context is irrelevant.

In the calculation, the photon is assumed to be located at the wave train head. In reality, because of QM-uncertainty, the photon collision can appear everywhere during the whole oscillation period (Heisenberg, 1930). This leads to QM uncertainty in the mirror and wave expansion, but in a statistical average the congruence between path and wave is true (see also Chap.5).

In the course of this process, matter, photons and space expand synchronously, mainly driven by the radiation pressure due to ULW photons. It should be mentioned that mirrors are in reality charges like baryons, leptons and fermions (from photon decay). The process has the potential to smooth out inhomogeneities and anisotropies in space and can be the reason why space looks so homogenous. However, the process leads to a steady expansion of space, which of course is not given in case of the so called inflation of space where within a time span between $10^{-36} \mathrm{~s}$ to $10^{-32} \mathrm{~s}$ the space expands by a factor of $10^{26}$. Concerning this theory see Guth (1997) and others.

In the wave description, the reflection theater uses the boundary condition that the component of the wave vector perpendicular to the perfectly reflecting mirrors is inversely proportional to the distance between them and that the parallel component can be neglected. The entire electric field has to be zero inside the perfect conductor, so that in particular the perpendicular component has to vanish at the boundary (mirror). At any rate, the wavelength would be approximately proportional to the distance. This would at least work for the lower-order standing wave modes. Since the photon momentum is basically the same as the wave vector (up to a factor $\hbar$ ), the photon momentum would then also decrease with increasing mirror distance, but not vanish.

The maximum expanded wavelength $\lambda$ assumed in this paper is on the order of $\tilde{\lambda}$, which is the diameter of space. In this context it should be mentioned that besides the zero point radiation, the isotropic radiation pressure due to the ULW photons also plays a decisive role in a model where mass is simulated by means of a photon that is confined in a cavity stabilized by this pressure (Schultheiss, 2013).

\section{Aspects Concerning the Conservation of Relativity}

Relativity fails during motion of charges/atoms against an isotropic flux of a short-wavelength photon-spectrum because of deceleration effects due to Doppler blue-shifts in front and red-shifts behind (Note deceleration or cooling of particles by means of surrounding light is in all aspects well investigated by Hänsch (1975) and Wineland (1975)). This is true for a short wavelength spectrum. However, according to results of Figure 2 and the comments, an instantaneous Doppler shift of ULW-photons can be neglected since the time span to execute a Doppler shift demands a full period oscillation of the ULW wave train, which takes about $\tilde{\lambda} / \mathrm{c} \approx 10^{17}$ seconds.

Besides the negligible Doppler shift, an increased collision frequency is expected if a charge moves with the velocity $\mathrm{v}$ against a light-fast ULW photon flux $\mathrm{j}$. This would also contradict to relativity since it causes a deceleration force. But this is not true. As already mentioned, because of the QM uncertainty $\Delta p \cdot \Delta x \geq \hbar$, the position $\Delta x$ of a photon with momentum $\Delta p=h / \tilde{\lambda}$ can be anywhere along the path of $\tilde{\lambda}$ (Heisenberg, 1930). In this view, the flux $\mathrm{j}$ consists of photons distributed everywhere between the wave head and the tail. An individual velocity of a charged particle does not give an ansatz to influence the results of QM-uncertainty.

\section{Estimation of the $\left(\omega / \omega_{p}\right)^{4}$-Value}

To describe gravitation, an intense flux of isotropic ULW-photons entering the interaction sphere of a charge is necessary. To give an estimate of the flux $\mathrm{j}$, one has to give an estimate of $\omega_{p}$ in Equation 5. In the reference Schultheiss (2008), a wide variation of $\omega / \omega_{p}$ between 1 and $10^{-9}$ is given. In Tab.1, a special parameter set $\omega / \omega_{\mathrm{p}} \approx 5 \cdot 10^{-6}$ which gives a flux of $\mathrm{j}=10^{45}$ photons/s is proposed. It turns out (see bottom line in Tab.1) that nearly $10^{20}$ photons enter the interaction sphere per elementary time $t_{0}=r_{0} / \mathrm{c}$. In addition, the energy density of the volume proportional to $r_{0}^{3}$ is of the order of the proton-neutron energy density, and finally the actual number of photons in this volume is of the order of $10^{40}$. This number (which will be called $\mathrm{u}$ ) reflects $\mathrm{u}=10^{40}, \sqrt{\mathrm{u}}=10^{20} ; \mathrm{u}$ is the ratio of the electrical and gravitational force between electron and proton in a hydrogen atom for arbitrary orbits $\mathrm{R}$ since both forces are proportional to $1 / \mathrm{R}^{2}$ and the radii cancel. 
Table 1. Dependence of the ULW photon flux $\mathrm{j}$, energy density $\varepsilon=j p / 4 \pi r_{p}^{2}$, where $\mathrm{p}$ is the momentum of the ULW photon $\left(\mathrm{p}_{\mathrm{ULW}} \approx 10^{-60} \mathrm{~kg} \cdot \mathrm{m} / \mathrm{s}\right)$ and interaction sphere radius $\mathrm{r}_{\mathrm{p}}$ as a function of disturbance frequency $\omega_{\mathrm{p}}$. The last line of the table corresponds to numbers which relate to elementary time and space (see text)

\begin{tabular}{|c|c|c|c|c|}
\hline$\omega / \omega_{\mathrm{p}}$ & $\mathbf{j}$ in photons $/ \mathbf{s}$ & $\mathbf{r}_{p}$ in $\mathbf{m}$ & Energy density $\varepsilon$ in $J / \mathrm{m}^{3}$ & ULW-photons $\mathbf{m}^{-3}$ \\
\hline $20 \cdot 10^{-6}$ & $6 \cdot 10^{42}$ & $4 \cdot 10^{-25}$ & $3 \cdot 10^{30}$ & \\
\hline $10 \cdot 10^{-6}$ & $10^{44}$ & $10^{-25}$ & $10^{33}$ & \\
\hline $5 \cdot 10^{-6}$ & $1,6 \cdot 10^{45}$ & $2.5 \cdot 10^{-26}$ & $10^{35}$ & $3 \cdot 10^{86}$ \\
\hline $3 \cdot 10^{-6}$ & $2.7 \cdot 10^{45}$ & $10^{-26}$ & $2 \cdot 10^{36}$ & \\
\hline $5 \cdot 10^{-6}$ & $6 \cdot 10^{19} / t_{0}$ & $2,5 \cdot 10^{-11} \mathrm{r}_{0}$ & $2 \cdot 10^{-10} \mathrm{~J} / \mathrm{r}_{0}{ }^{3}$ & $3 \cdot 10^{41} / \mathrm{r}_{0}{ }^{3}$ \\
\hline
\end{tabular}

Note that the flux of ULW photons must have the same value everywhere in space. However each photon -- or respectively: each wave -- measures the periodic frequency $\omega_{\text {Star }}$ of every interaction partner. Hereby, the periodic motion $\omega_{\text {Star }}$ will be interpreted to be the natural frequency, that is, $\omega_{\text {Star }}=\omega_{\mathrm{p}}$. If it is small $\left(\omega_{\text {Star }}=\omega_{p} \rightarrow \omega\right)$, then the cross section is large and the probability of a reflection increases. A ULW-photon that crosses a galaxy disk tends to interact more with outer stars since the angular velocity there is lower than is the case for inner stars. Formally one can understand this as the dependence of the gravitational constant $\mathrm{g}$ (or better factor) on the angular frequency $\omega_{p}$. Equation 5 gives:

$$
g\left(\omega_{p}\right)=\frac{4}{3} \sigma j \frac{h r_{0}^{2}}{\lambda m_{p}^{2}} \frac{\omega^{4}}{\omega_{p}^{4}}
$$

To discuss Equation 8: In comparison to the standard value of the gravitation constant, which is $6,67 . .10^{-11}$ $\mathrm{m}^{3} /\left(\mathrm{kg} \cdot \mathrm{s}^{2}\right)$, the pre-factor in Equation 8 already gives a measure of the maximum gravitation factor, which is on the order of $10^{12} \mathrm{~m}^{3} /\left(\mathrm{kg} \cdot \mathrm{s}^{2}\right)$.

Now we can make a correlation between frequencies and distances in space. With $\tilde{\lambda}$, which is roughly the diameter of space, we state that:

$$
\omega=\frac{2 \pi \mathrm{c}}{\tilde{\lambda}}
$$

Analogously, we determine the characteristic length $\Lambda_{\mathrm{p}} \operatorname{using} \omega_{p}$ :

$$
\omega_{\mathrm{p}}=\frac{2 \pi \mathrm{c}}{\Lambda_{\mathrm{p}}}
$$

and finally the minimum gravitation factor $g_{p}$, using the abbreviation $\omega / \omega_{\mathrm{p}}=\Omega$, has the form

$$
g_{p}=\text { const } \frac{\Lambda_{p}^{4}}{\tilde{\lambda}^{4}}=\text { const } \Omega^{4} .
$$

Now we can see that an increase of the minimum length $\Lambda_{\mathrm{p}}$ by $\Delta$ will cause an increase of the gravitational factor to $g_{p}(\Delta)$.

$$
g_{p}(\Delta)=\operatorname{const} \frac{(\tilde{\lambda} \Omega+\Delta)^{4}}{\tilde{\lambda}^{4}}=\operatorname{const} \Omega^{4}\left(1+\frac{\Delta}{\tilde{\lambda} \Omega}\right)^{4}=g_{\min }\left(1+\frac{\Delta}{\tilde{\lambda} \Omega}\right)^{4}
$$

\section{Spectrum of Ultralong-Wavelength Photons can Simulate Dark Matter-Like Effects in Spiral Galaxies}

The theoretical orbital velocity $v$ of a star in distance $r$ from the center of its galaxy is determined by the so-called Kepler condition, where $\mathrm{m}$ is the mass of a star and $\mathrm{M}$ is the mass of all visible stars within the star's orbital:

$$
g \cdot \frac{m \cdot M(r)}{r^{2}}=\frac{m \cdot v^{2}}{r}
$$

The orbital velocity v is (see Figure 3 green curve): 


$$
v(r)=\sqrt{\frac{g \cdot M_{\text {visible }}(r)}{r}}
$$

Since (Sofue \& Rubin, 2001; Sofue, 2013) shows that the outer branches of nearly all spiral galaxies rotate with constant higher velocity (see, for example, the black curve in Figure 3), the ansatz for the action of the dark matter $\mathrm{M}_{\mathrm{dark}}$ is:

$$
v\left(r, M_{\text {dark }}\right)=\sqrt{\frac{g \cdot\left[M_{\text {visible }}(r)+M_{\text {dark }}(r)\right]}{r}}
$$

The orbital velocity of the outer branches is nearly constant while for $\mathrm{M}_{\text {visible }}$ it decreases with increasing radius. The lack of gravitating mass must be compensated by the increasing dark matter $M_{\text {dark }}(r)$ distributed in the outer sphere; this is simply assumed, and has no other function. In this paper we take Equation 15 and use $\mathrm{g}_{\mathrm{p}}(\Delta)$ instead of $\mathrm{g}$ :

$$
v(r, \Delta)=\sqrt{\frac{g_{p}(\Delta) \cdot M_{\text {visible }}(r)}{r}}
$$

Finally, using Equation 12 we have:

$$
v(r, \Delta)=\left(1+\frac{\Delta}{\tilde{\lambda} \Omega}\right)^{2} \cdot \sqrt{\frac{g_{p} \cdot M_{\text {visible }}(r)}{r}} .
$$

We come back to the numerical estimates in Table 1 where we assume that $\Omega=5 \cdot 10^{-6}$ and ULW-photons with wavelengths of the order of the diameter of space (about $10^{26} \mathrm{~m}$; Note: $\Delta$ and $\mathrm{r}$ in Equation 17 are identically). Thus, the characteristic length $\Lambda_{\mathrm{p}}=\tilde{\lambda} \cdot \Omega$ (see Equation 11) is on the order of $5 \cdot 10^{20} \mathrm{~m}$ or $15 \mathrm{kpc}$. In this case the pre-factor in Equation 17 at the distance of $15 \mathrm{kpc}$ is approximately 4 . As one can see, this is roughly the difference between the green Kepler and the red $v(r, \Delta)$-curve in Figure 3 at this point (M33 galaxy, see below).

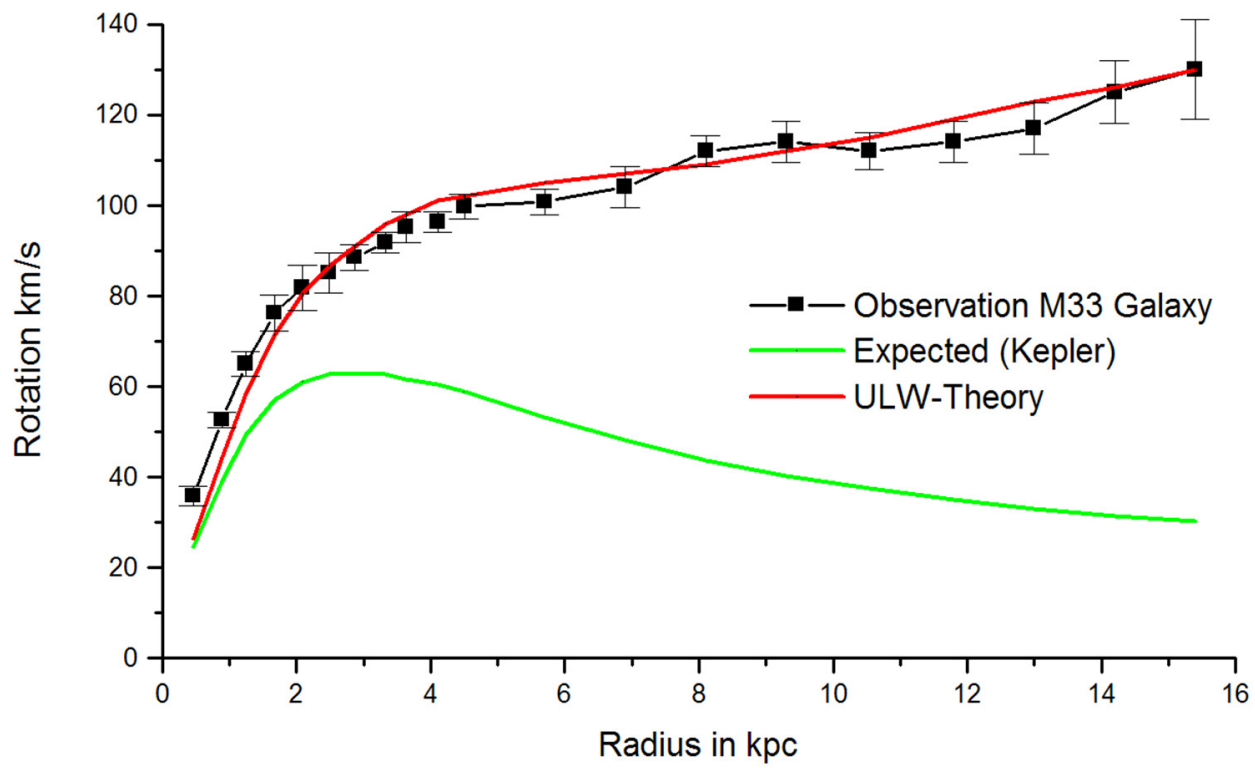

Figure 3 Rotation anomaly of stars of the M33 spiral galaxy as a function of radius (Corbelli \& Salucci, (2000)) . Instead of slowing down, the rotation velocity increases steadily with increasing radius (black data points with error bars). This contradicts the expected rotation behavior predicted by the gravitation of visible stars (green curve). The ULW mechanism predicts an increased gravitational factor with increasing radius because $\omega_{p}$ decreases (red curve)

In the following, the theory will be tested with the well-investigated M33 galaxy (Corbelli \& Salucci, 2000). In Figure 3, the black data points with error bars (connected with eye leading lines) show the observations of the 
rotation velocity. The data are obtained by means of the measured red-shift of visible light from stars and the shift of the $21 \mathrm{~cm}-\mathrm{H}_{2}$-line (Richter \& Valentijn, 1999) of the far-distant outer region. Since the passage of light through hydrogen, either atomic or molecular, is always slightly inelastic, the nature of the red shift is may be not purely Doppler-based. The green curve indicates the expected Kepler rotation (Equation 14) on the basis of the visible mass. These data show clearly the discrepancy between the expectation of Newton's theory and the observation at the outer branches. Finally the red curve shows the effect of a variable gravitation constant on the basis of the wave structure of the ULW photons with Rayleigh scattering. The agreement of the curve at large distances is very good. Minor deviations can be seen at smaller distances (less than $1 \mathrm{kpc}$ ). Comparison with the data indicates that the model may correctly describe the physics.

\section{Conclusion}

This investigation supports the assumption that gravity may have electromagnetic roots. The understanding of the rotation anomaly of outer stars in galaxies seems to be based on the characteristic $\left(\omega / \omega_{p}\right)^{4}$-dependence of Rayleigh scattering of electromagnetic waves with charged particles (blueness of sky, see Equation 1). At lower $\omega_{p}$, that is, at increasing path distances (see Equation 17), the cross section for scattering and the gravitation factor increase. The remarkable accuracy of the theoretical curve in Figure 3 is based on the assumption of an $\Omega$-value of $5 \cdot 10^{-6}$. This value reflects an energy density of the isotropic ULW photon-flux comparable to the proton energy density. The ULW-photon-flux can be understood as a result of the more or less similar expansion of the particle path and the photon wavelength after the Big Bang. Furthermore, factors such as $10^{40}$ and $10^{20}$ appear, which may indicate important, but not yet understood physical connections.

It should be emphasized again that this theory avoids introducing new physics such as dark matter and dark energy. To achieve this result, basic assumptions with far-reaching consequences must be made. But this can be done on the basis of well understood physics.

\section{Acknowledgement}

I would like to express my gratitude to Edith Borie, who supported the development of the paper through fruitful discussions and assisted during the final editing. I also want to thank Oliver C. Schultheiss for suggestions during the final reading.

\section{References}

Aronson, S. (1964). The gravitational theory of Georges-Louis Le Sage. The Natural Philosopher, 3, 51.

Bergstrom, L. (2000). Non-baryonic dark matter: Observational evidence and detection methods. Reports on Progress in Physics, 63(5), 793-841. http://dx.doi.org/10.1088/0034-4885/63/5/2r3

Casimir, H. B. G. (1948), On the attraction between two perfectly conducting plates. Koinkl. Ned. Akyd. Wetenshap. Proc., 51, 793

Corbelli, E., \& Salucci, P. (2000). The extended rotation curve and the dark matter halo of M33. MNRAS, 311, 441.

Einstein, A. (1916). Relativity: The Special and General Theory (Translation 1920). New York: H. Holt and Company.

Feynman, R. P. (1965). Lectures on Physics, California Institute of Technology (Vol. 1, pp. 32-38) (Rayleigh Scattering).

Freeman, K. C. (1970). Astrophys. J. 160, 881. In M. Persic, \& P. Salucci (Eds.), Dark and visible matter in Galaxies. ASP.

Fujita, T., \& Kanda, N. (2011). A Proposal to Measure Photon-Photon Scattering. arXiv: 1106.0465v1 [hep-ph] 30 May 2011.

Gott III, J. R., Jurić, M., Schlegel, D., Hoyle, F., Vogeley, M., Tegmark, M., ... \& Brinkmann, J. (2005). A Map of the Universe. The Astrophysical Journal, 624(2), 463.

Guth, A. (1997). The Inflationary Universe: The Quest for a New Theory of Cosmic Origins. Basic Books (pp. 233-234).

Hänsch, T. W., \& Shawlow, A. L. (1975). Cooling of Gases by Laser Radiation. Optics Communications, 13, 68 arXiv: 1106.0465v1 [hep-ph] 30 May 2011. http://dx.doi.org/10.1016/0030-4018(75)90159-5

Heisenberg, W. (1930). The Physical Principles of the Quantum Theory. In C. Eckart, \& F.C. D. Hoyt (Trans.). (1949). 
Kanda, N. (2011). Light-Light Scattering. arXiv 1106.0592v1.

Klein,M.J., Kox, A.J., \& Schulmann, R. (1996). The Collected Papers of Albert Einstein. Princton University Press (Vol.3. DOC 23, 497, footnote [11]).

Lemaitre, A. G. (1931). A homogeneous universe of constant mass and increasing radius accounting for the radial velocity of extra-galactic nebulae. Monthly Notices of the Royal Astronomical Society, 91(5), 483-490.

Reid, M. J., Readhead, A. C. S., Vermeulen, R. C., \& Treuhaft, R. N. (1999). The proper motion of Sagittarius A*. I. First VLBA results. The Astrophysical Journal, 524(2), 816-823. http://dx.doi.org/10.1086/307855

Richter, P., de Boer, K. S., Widmann, H., Kappelmann, N., Gringel, W., Grewing, M., \& Barnstedt, J. (1999). Discovery of molecular hydrogen in a high-velocity cloud of the Galactic halo. Nature, 402(6760), 386-387.

Schultheiss C. (2008), Mass Attraction Caused by Ultralong-Wave Photons? Adv. Studies Theor. Phys., 2(10), 491-505.

Schultheiss C. (2013), Momentum and Energy of a Mass Consisting of Confined Photons and Resulting Quantum Mechanical Implications. Adv. Studies Theor. Phys., 7(12), 555-583.

Sofue, Y. (2013). Mass Distribution and Rotation Curve in the Galaxy. In G. Gilmore (Ed.), Planets, Stars and Stellar Systems (pp. 985-1037). Springer Netherlands.

Sofue, Y., \& Rubin, V. (2001). Review on RCs: Rotation Curves of Spiral Galaxies. ARAA, 39, 137-174.

Valentijn, E. A., \& van der Werf, P. P. (1999). First extragalactic direct detection of large-scale molecular hydrogen in the disk of NGC 891. The Astrophysical Journal Letters, 522(1), L29-35.

Wineland, D. J., \& Dehmelt, H. (1975). Proposed $10^{14} \Delta v<v$ Laser Fluorescence Spectroscopy on T1+Mono-Ion Oscillator III" (PDF). Bulletin of the American Physical Society, $20,637$.

\section{Copyrights}

Copyright for this article is retained by the author(s), with first publication rights granted to the journal.

This is an open-access article distributed under the terms and conditions of the Creative Commons Attribution license (http://creativecommons.org/licenses/by/3.0/). 